\title{
Predicted Electronic and Thermodynamic Properties of a Newly Discovered $\mathrm{Zn}_{8} \mathrm{Sb}_{7}$ Phase
}

\author{
Gregory S. Pomrehn, ${ }^{*}$ Eric S. Toberer, ${ }^{\dagger}$ G. Jeffrey Snyder, and Axel van de Walle \\ Materials Science, California Institute of Technology, 1200 East California Boulevard, Pasadena, California 91125, United States
}

ABSTRACT: A new binary compound, $\mathrm{Zn}_{8} \mathrm{Sb}_{7}$, has recently been prepared in nanoparticulate form via solution synthesis. No such phase is known in the bulk phase diagram; instead, one would expect phase separation to the good thermoelectric semiconductors $\mathrm{ZnSb}$ and $\mathrm{Zn}_{4} \mathrm{Sb}_{3}$. Here, density functional calculations are employed to determine the free energies of formation, including effects from vibrations and configurational disorder, of the relevant phases, yielding insight into the phase stability of $\mathrm{Zn}_{8} \mathrm{Sb}_{7}$. Band structure calculations predict $\mathrm{Zn}_{8} \mathrm{Sb}_{7}$, much like $\mathrm{ZnSb}$ and $\mathrm{Zn}_{4} \mathrm{Sb}_{3}$, to be an intermetallic semiconductor with similar thermoelectric properties. If sufficient entropy or surface energy exists to stabilize the bulk material, it would be stable in a limited temperature window at high temperature.

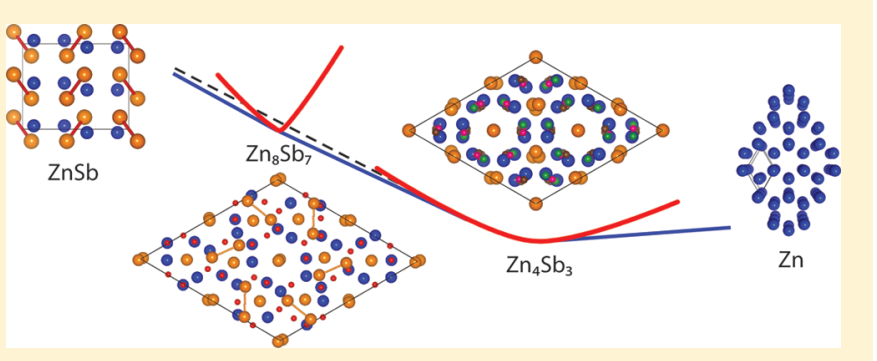

\section{INTRODUCTION}

Intermetallic compounds are fascinating due to the diversity of their crystal structures and resulting properties. For thermoelectric applications, $\mathrm{ZnSb}$ and $\mathrm{Zn}_{4} \mathrm{Sb}_{3}$ are two intermetallic compounds which exhibit good thermoelectric efficiencies. In particular, $\mathrm{Zn}_{4} \mathrm{Sb}_{3}$ has a thermoelectric figure of merit $(z T)$ in excess of unity ${ }^{1,2}$ due to its low thermal conductivity, which arises in part due to its complex, defect-ridden structure. Both of these materials have band gaps at or near the Fermi level which can be rationalized from valence charge counting. ${ }^{3}$

Recently, a new binary $\mathrm{Zn}-\mathrm{Sb}$ phase between $\mathrm{ZnSb}$ and $\mathrm{Zn}_{4} \mathrm{Sb}_{3}$ was discovered and characterized at room temperature by Birkel et al. ${ }^{4}$ Binary nanoparticles were synthesized in solution through the controlled reaction of elemental $\mathrm{Zn}$ and $\mathrm{Sb}$ nanoparticles. The small particle size of the reactants ensures minimum diffusion paths, low activation barriers, and low reaction temperatures, thereby eliminating solid-solid diffusion as the rate-limiting step as found in conventional bulk-scale solid-state synthesis. In the present work, the authors denote this new phase as $\mathrm{Zn}_{8} \mathrm{Sb}_{7}$, based on the idealized crystal structure, rather than $\mathrm{Zn}_{1+\delta} \mathrm{Sb}$ (with $\delta=0.068$ ) as used in ref 4 .

Determination of the $\mathrm{Zn}_{8} \mathrm{Sb}_{7}$ structure was a tour de force of automated electron diffraction tomography (ADT) combined with precession electron diffraction (PED) on individual nanoparticle grains. This work yielded the structure in Figure 1, shown along the [001] and [100] directions in the distorted hexagonal cell $(P \overline{1})$. Much like $\mathrm{Zn}_{4} \mathrm{Sb}_{3}, \mathrm{Zn}_{8} \mathrm{Sb}_{7}$ contains both $\mathrm{Sb}_{2}{ }^{4-}$ dimers and isolated $\mathrm{Sb}^{3-}$; however, the long-range atomic arrangement is completely distinct.

The $\mathrm{Zn}_{8} \mathrm{Sb}_{7}$ phase enters into an interesting region of the binary phase diagram ${ }^{5}$ between $\mathrm{ZnSb}$ and $\mathrm{Zn}_{4} \mathrm{Sb}_{3}$. Since the three phases are so close in composition, the competition for thermodynamic stability will be fierce. Below $\sim 250 \mathrm{~K}, \mathrm{Zn}_{4} \mathrm{Sb}_{3}$ transforms to a variant (the $\alpha$ or $\alpha^{\prime}$ phase) where the interstitial atoms form an ordered arrangement. ${ }^{6,7}$ The disordered $\beta$ phase is stable from $\sim 250 \mathrm{~K}$ (to $700 \mathrm{~K}$ ), while no compounds have been characterized in the bulk at compositions between $\mathrm{Zn}_{4} \mathrm{Sb}_{3}$ and $\mathrm{ZnSb}$. Several phase diagrams have been reported that include a phase between $\mathrm{Zn}_{4} \mathrm{Sb}_{3}$ and $\mathrm{ZnSb}$ stable only at high temperature, ${ }^{5,8,9}$ sometimes denoted as $\gamma$, but no crystal structures have been determined for these high-temperature phases.

$A b$ initio calculations have considered various interstitial configurations of $\mathrm{Zn}_{4} \mathrm{Sb}_{3}$ at $0 \mathrm{~K}$ and found that they should decompose to $\mathrm{ZnSb}$ and $\mathrm{Zn}$ when considering the enthalpy of formation alone. ${ }^{10-13}$ Additional theoretical work has recently shown $\mathrm{Zn}_{4} \mathrm{Sb}_{3}$ to be entropically stabilized due to the high configurational disorder with the three interstitial $\mathrm{Zn}$ sites and the effects of lattice vibrations. ${ }^{14}$ The narrow stable composition range is near $\mathrm{Zn}_{3.898} \mathrm{Sb}_{3}$, as inferred from hall carrier measurements and valence charge counting, ${ }^{2,14}$ which is off from the valence-balanced composition of $\mathrm{Zn}_{3.9} \mathrm{Sb}_{3}$. We will continue to use the conventional $\mathrm{Zn}_{4} \mathrm{Sb}_{3}$ terminology.

In this work, we explore the electronic and thermodynamic properties of a theoretical bulk $\mathrm{Zn}_{8} \mathrm{Sb}_{7}$ phase. Electronic structure calculations probe the band structure and valence band edge character of $\mathrm{Zn}_{8} \mathrm{Sb}_{7}$ to compare with known bulk thermoelectrics $\mathrm{ZnSb}$ and $\mathrm{Zn}_{4} \mathrm{Sb}_{3}$. Since the formation of $\mathrm{Zn}_{8} \mathrm{Sb}_{7}$ is not readily observed in practice, a thermodynamic investigation determines if the phase might be thermodynamically stable or metastable with respect to competing $\mathrm{Zn}-\mathrm{Sb}$ phases. A thorough thermodynamic treatment considers the bonding enthalpy as well as possible sources of entropy such as configurational disorder and

Received: March 18, 2011

Published: June 16, 2011 


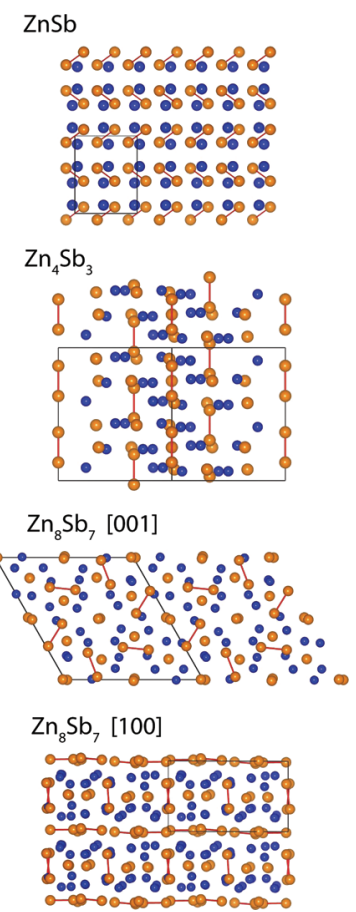

Figure 1. Structures of the known room-temperature zinc antimonide phases, shown with zinc in blue, $\mathrm{Sb}_{2}{ }^{4-}$ dimers and isolated $\mathrm{Sb}^{3-}$ in orange. The anionic structure of $\mathrm{ZnSb}$ is exclusively $\mathrm{Sb}_{2}{ }^{4-}$ dimers, while $\mathrm{Zn}_{4} \mathrm{Sb}_{3}$ and $\mathrm{Zn}_{8} \mathrm{Sb}_{7}$ contain both $\mathrm{Sb}^{3-}$ and $\mathrm{Sb}_{2}{ }^{4-}$ moieties.

atomic vibrations. The results will guide future experiments to synthesize bulk $\mathrm{Zn}_{8} \mathrm{Sb}_{7}$.

\section{COMPUTATIONAL METHODS}

To account for the configurational disorder present in many $\mathrm{Zn}-\mathrm{Sb}$ phases, a thermodynamic ensemble approach is employed using data from first-principles calculations. A Grand Canonical Potential ${ }^{15}$ (GCP) is formulated for each phase according to

$$
\phi(T, \boldsymbol{\mu})=-\frac{k_{\mathrm{B}} T}{N} \ln \left(\sum_{s} \mathrm{e}^{-\left(E_{s}-\boldsymbol{\mu} \cdot \mathrm{N}_{s}\right) / k_{\mathrm{B}} T}\right)
$$

where $k_{\mathrm{B}}$ is Boltzmann's constant, $T$ is temperature, $N$ is the total number of atoms in the system, while, for a given state $s, E_{s}$ is the total energy of state, $\mathbf{N}_{s}$ is a vector containing the number of atoms for each chemical species (with elements summing to $N$ ), and $\boldsymbol{\mu}$ is a vector containing the chemical potential of each species. Since $\mathrm{ZnSb}$ and $\mathrm{Zn}$ phases exhibit relatively low configurational disorder, the GCP greatly simplifies:

$$
\phi(T, \boldsymbol{\mu})=\frac{\varepsilon_{0}}{n}-\boldsymbol{\mu} \cdot \mathbf{x}_{0}
$$

where $\varepsilon_{0}$ is the energy per unit cell, $n$ is the number of atoms per unit cell, and $\mathbf{x}_{\mathbf{0}}$ is a vector of the atomic fraction of each species.

For $\mathrm{Zn}_{8} \mathrm{Sb}_{7}$ and $\mathrm{Zn}_{4} \mathrm{Sb}_{3}$, we factor the GCP according to an "independent cells" approximation. We assume that each primitive unit cell is non-interacting with neighboring cells, in the sense that the defect configuration present in one cell does not affect the energies of defect configurations in a nearby cell. (All our $a b$ initio calculations are nevertheless performed on infinite periodic systems with suitable $k$ point sampling.) This approach is useful in these systems because the unit cells are rather large. This assumption is validated by computing the energy of supercells with different configurations in each primitive unit cell and comparing it to that predicted by summing the energies of the constituent primitive cells. Under the independent cells approximation,

$$
\phi(T, \boldsymbol{\mu})=\frac{\varepsilon_{0}}{n}-\boldsymbol{\mu} \cdot \mathbf{x}_{0}-\frac{k_{\mathrm{B}} T}{n} \ln \left(1+\sum_{i>0} m_{i} \mathrm{e}^{-\left(\Delta \varepsilon_{i}-\boldsymbol{\mu} \cdot \Delta \mathbf{n}_{i}\right) / k_{\mathrm{B}} T}\right)
$$

where $\varepsilon_{0}$ is the ground-state energy per unit cell, $n$ is the number of atoms per unit cell, and $\mathbf{x}_{\mathbf{0}}$ is the ground-state composition. For each configuration $i, m_{i}$ is the symmetric multiplicity, $\Delta \varepsilon_{i}$ is the change in energy from the ground state, and $\Delta \mathbf{n}_{i}$ is the change in the number of atoms from the ground state.

The total energy of each atomic configuration was calculated using Density Functional Theory (DFT) with the PBE exchange-correlation functional. ${ }^{16}$ Calculations utilized the projector augmented wave (PAW) method ${ }^{17}$ as implemented in VASP, ${ }^{18}$ neglecting spin-orbit coupling. All unit cell parameters and atomic positions were allowed to relax to find the lowest energy arrangement to within $10^{-4} \mathrm{eV}$ per primitive unit cell. A final static calculation was performed for an accurate total energy. Energy convergence with respect to $k$-points was achieved with a $\Gamma$-centered grid with $14 k$-points in the irreducible Brillouin zone.

Configurational arrangements for $\mathrm{Zn}_{4} \mathrm{Sb}_{3}$ were enumerated by considering all possible interstitial combinations on known $\mathrm{Zn}$ interstitial sites. ${ }^{3,14}$ Configurational arrangements of $\mathrm{Zn}_{8} \mathrm{Sb}_{7}$ are much more difficult to enumerate appropriately without guidance from experimentally known defect lattice sites. The configurational disorder then was probed by a systematic enumeration of new possible configurations on the $\mathrm{Zn}$ sublattice. The specific enumeration process is described later in the Results and Discussion. Configurations with $\mathrm{Zn}$ vacancies and interstitials deviating from the $\mathrm{Zn}_{8} \mathrm{Sb}_{7}$ composition are also considered. DFT calculations were performed on select $\mathrm{Zn}_{8} \mathrm{Sb}_{7}$ configurations since it would be computationally prohibitive to calculate all possibilities. Atomic positions were allowed to relax to a local minimum and were verified to not have settled into identical configurations. While this method is by no means exhaustive in gauging the configurational disorder in $\mathrm{Zn}_{8} \mathrm{Sb}_{7}$, it does provide a basis for making certain assumptions, to follow in the Results and Discussion.

Phonon density of states and vibrational free energies were calculated using the "supercell" method as implemented in the Alloy Theoretic Automated Toolkit (ATAT). ${ }^{19-21}$ The computational resources needed to compute phonon modes for all $\mathrm{Zn}_{8} \mathrm{Sb}_{7}$ configurations are prohibitive (360 perturbation structures per configuration per volume), so the phonon modes were only calculated for the nominal configuration. In all, phonon modes were calculated for one $\mathrm{Zn}_{8} \mathrm{Sb}_{7}$ configuration and several $\mathrm{Zn}_{4} \mathrm{Sb}_{3}$ configurations as well as end-members $\mathrm{Zn}$ and $\mathrm{ZnSb}$ at $0 \%, 1 \%$, and $2 \%$ strain to account for the effects of thermal expansion under the quasi-harmonic approximation.

The vibrational contribution to the free energy is incorporated into the GCP through a nested sum in the partition function. ${ }^{22}$ For each distinct configuration, phonon occupation accounts for small displacements around the local energy minimum, resulting in a temperaturedependent free energy correction.

GCPs were assembled for $\mathrm{Zn}, \mathrm{Zn}_{4} \mathrm{Sb}_{3}, \mathrm{Zn}_{8} \mathrm{Sb}_{7}$, and $\mathrm{ZnSb}$. Phase equilibrium is determined by equality of two respective GCPs. The equilibrium composition of each phase can be determined by

$$
\nabla_{\boldsymbol{\mu}} \phi(T, \boldsymbol{\mu})=-\mathbf{x}
$$

and the free energy is calculated as

$$
f(T, \mathbf{x})=\phi\left(T, \boldsymbol{\mu}_{\mathbf{x}}\right)+\boldsymbol{\mu}_{\mathbf{x}} \cdot \mathbf{x}
$$

where $\mu_{\mathbf{x}}$ is the chemical potential that stabilizes the phase at composition $\mathbf{x}$.

The Seebeck coefficient is calculated for the ground-state structures using the Boltzmann transport equation within the constant relaxation 


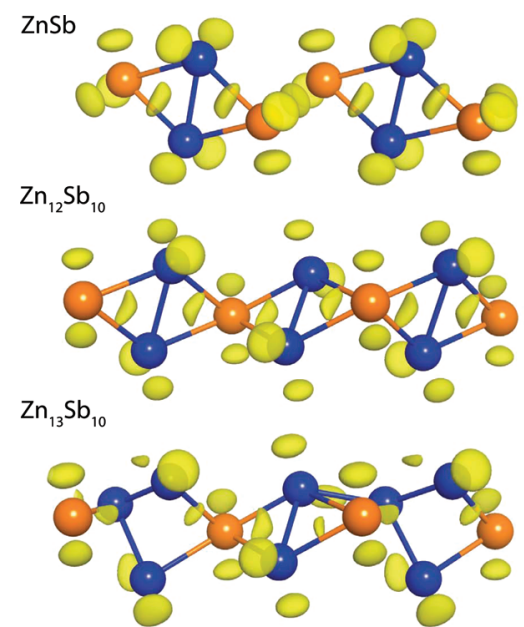

$\mathrm{Zn}_{8} \mathrm{Sb}_{7}$
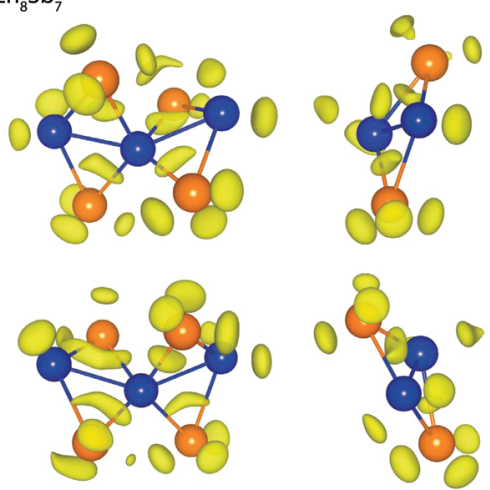

Figure 2. Electron density difference plots showing networked structures of diamond moieties of $\mathrm{ZnSb}, \mathrm{Zn}_{4} \mathrm{Sb}_{3}$, and $\mathrm{Zn}_{8} \mathrm{Sb}_{7}$. Yellow isosurfaces show regions of electron accumulation with respect to unperturbed overlapping atomic wave functions. In all structures, $\mathrm{Sb}$ atoms share a local maximum "banana" with two closely spaced $\mathrm{Zn}$ atoms, forming diamond-shaped moieties. The network of diamonds becomes increasingly complex in $\mathrm{Zn}_{4} \mathrm{Sb}_{3}$ and $\mathrm{Zn}_{8} \mathrm{Sb}_{7}$, providing a rich source of entropy.

time approximation as implemented in the BoltzTraP code. ${ }^{23}$ A dense $k$ point mesh is required to evaluate the Fermi integrals over the entire Brillouin zone. Convergence of the Seebeck coefficient with respect to carrier concentration is achieved in $\mathrm{Zn}_{8} \mathrm{Sb}_{7}$ with a $14 \times 14 \times 14$ $\Gamma$-centered $k$-point grid. For $\mathrm{Zn}_{4} \mathrm{Sb}_{3}$ and $\mathrm{ZnSb}$ structures, $16 \times 16 \times 16$ and $40 \times 32 \times 32 k$-point grids are used, respectively.

\section{RESULTS AND DISCUSSION}

Crystal Structure and Charge Counting. The $\mathrm{Zn}-\mathrm{Sb}$ structures in Figure 1 can all be described using the Zintl-Klemm formalism, ${ }^{24}$ in which $\mathrm{Zn}$ cations donate electrons to $\mathrm{Sb}$ anions which may then form covalent bonds with other $\mathrm{Sb}$ to complete their valence shells. A molecular orbital (MO) approach, which includes interactions between $\mathrm{Zn}$ and $\mathrm{Sb}$, can also successfully rationalize the electron count. ${ }^{13} \mathrm{In} \mathrm{ZnSb}(\mathrm{Pbca})$, all the respective $\mathrm{Zn}$ and $\mathrm{Sb}$ atoms are symmetrically equivalent. The $\mathrm{Zn}$ cations each donate two electrons to an $\mathrm{Sb}$ anion, and the $\mathrm{Sb}$ atoms form $\mathrm{Sb}_{2}{ }^{4-}$ dimers. $\mathrm{A}$ valence-precise structure results with $8 \mathrm{Zn}^{2+}$ and $4 \mathrm{Sb}_{2}{ }^{4-}$ per unit cell. One then can rationalize why $\mathrm{ZnSb}$ behaves electronically as an intrinsic semiconductor with a band gap between the valence band (dominated by $\mathrm{Sb}$

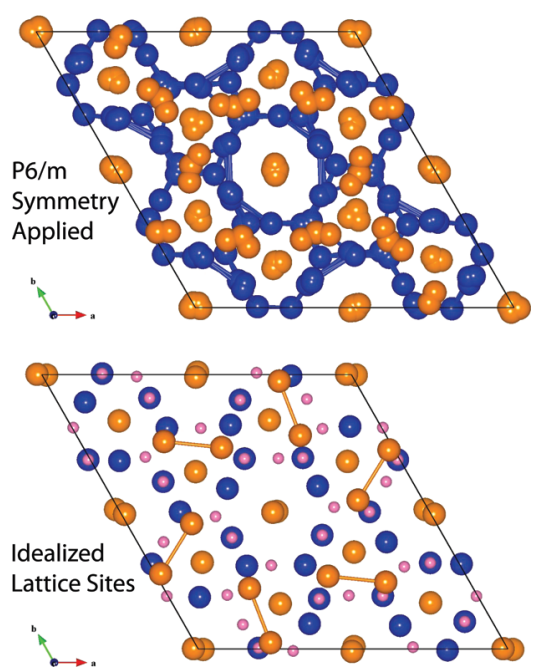

Figure 3. (Top) $\mathrm{Zn}_{8} \mathrm{Sb}_{7}$ lattice sites after applying all 12 symmetry operations of the $P 6 / m$ space group. The orange $\mathrm{Sb}$ sites map relatively closely to the original sites. Many of the blue $\mathrm{Zn}$ sites map to new sites, while a few map to nearly symmetrically equivalent sites. (Bottom) Idealized lattice sites after removing nearly equivalent sites. The original $28 \mathrm{Sb}$ sites and $32 \mathrm{Zn}$ sites are in orange and blue, respectively. An additional 66 unique $\mathrm{Zn}$ interstitial sites are shown in pink.

p-states) and the conduction band (composed primarily of $\mathrm{Zn}$ s-states).

The $\mathrm{Zn}_{4} \mathrm{Sb}_{3}(R \overline{3} c)$ framework structure is composed of $30 \mathrm{Sb}$ (orange sites in Figure 1) and $36 \mathrm{Zn}$ (blue sites) in the conventional unit cell. The anionic framework is divided between $6 \mathrm{Sb}_{2}{ }^{4-}$ dimers and 18 isolated $\mathrm{Sb}^{3-}$. A fully occupied cation framework of $36 \mathrm{Zn}^{2+}$ is insufficient to satisfy a valence charge balance. Three additional $\mathrm{Zn}^{2+}$ are needed to satisfy the charge balance at a composition of $\mathrm{Zn}_{13} \mathrm{Sb}_{10}$. These additional $\mathrm{Zn}$ atoms can occupy any of three symmetrically distinct regions for interstitial sites (pink, green, and brown sites), introducing configurational disorder not present in $\mathrm{ZnSb}$. Thermodynamically, $\mathrm{Zn}_{4} \mathrm{Sb}_{3}$ is stable only in a narrow range of composition near $\mathrm{Zn}_{12.992} \mathrm{Sb}_{10}$, which is slightly valence deficient. This explains the persistent p-type doping observed experimentally in $\mathrm{Zn}_{4} \mathrm{Sb}_{3}$.

Examining the $\mathrm{Zn}_{8} \mathrm{Sb}_{7}(P \overline{1})$ structure reveals some similarities to $\mathrm{Zn}_{4} \mathrm{Sb}_{3}$. The anionic $\mathrm{Sb}$ framework is only slightly distorted from a high-symmetry structure that can be described with a $\mathrm{P} 6 / \mathrm{m}$ space group. There are $10 \mathrm{Sb}_{2}{ }^{4-}$ dimers and 8 isolated $\mathrm{Sb}^{3-}$, all sitting close to points of symmetry. The cation $\mathrm{Zn}$ framework exhibits low symmetry with $32 \mathrm{Zn}^{2+}$. This satisfies a valenceprecise condition, suggesting that $\mathrm{Zn}_{8} \mathrm{Sb}_{7}$, like $\mathrm{ZnSb}$ and $\mathrm{Zn}_{13} \mathrm{Sb}_{10}$, should behave as an intrinsic semiconductor.

We further investigate the structure of $\mathrm{Zn}_{8} \mathrm{Sb}_{7}$ with the help of electron density difference (EDD) plots. The EDD compares the calculated electron charge density with the charge density resulting from the overlap of non-interacting single-atom electronic wave functions. EDD plots thus reveal where charge accumulation and depletion occur as atomic wave functions interact. Charge accumulation between atoms suggests the presence of a covalent bond.

Figure 2 shows the EDD around a diamond moiety in $\mathrm{ZnSb}$, which clearly reveals charge accumulation lobes in a tetrahedral arrangement around each $\mathrm{Sb}$ atom. In agreement with a previous molecular orbital study, ${ }^{13}$ one lobe of each $\mathrm{Sb}$ is shared between two closely spaced $\mathrm{Zn}$ atoms, forming a repeated diamond unit. 

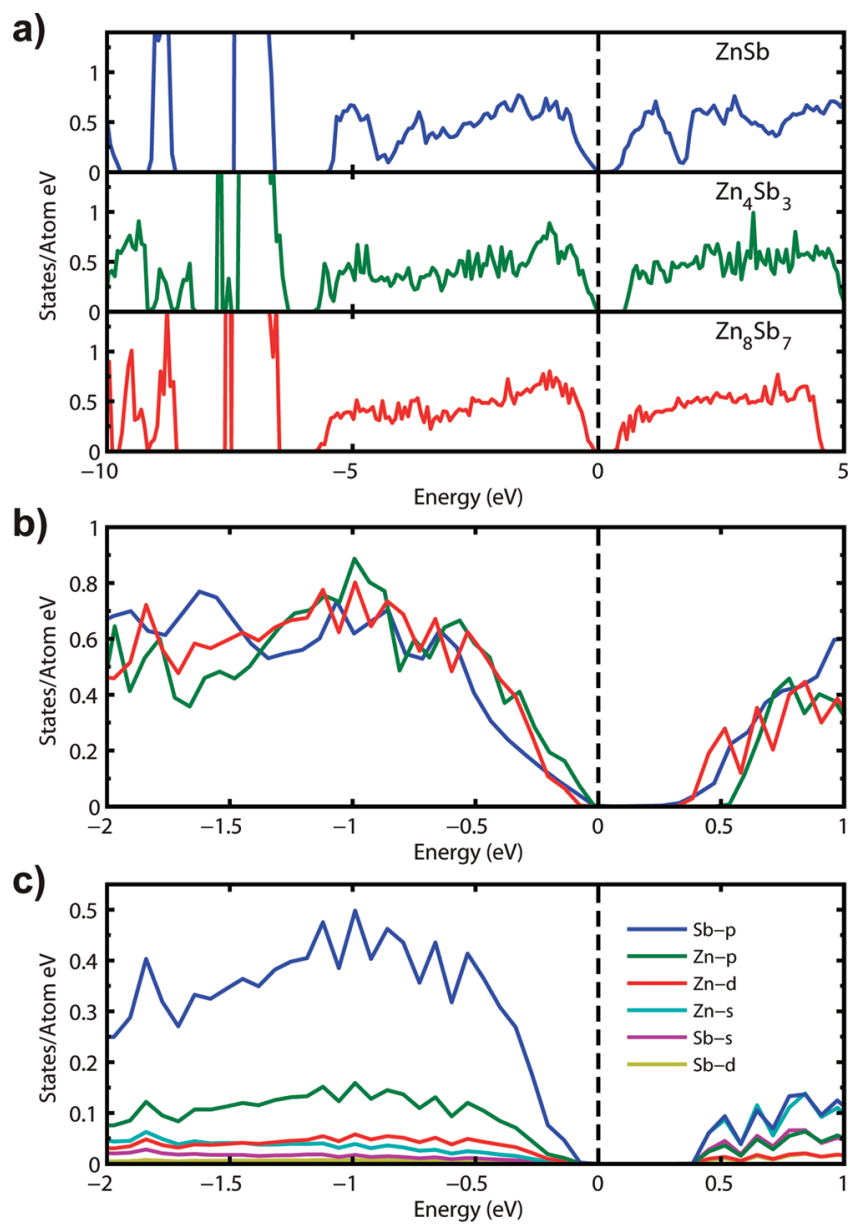

Figure 4. (a) Calculated density of states for of $\mathrm{ZnSb}, \mathrm{Zn}_{4} \mathrm{Sb}_{3}$, and $\mathrm{Zn}_{8} \mathrm{Sb}_{7}$. (b) Comparison near the Fermi level and (c) atomic orbital projected density of states for $\mathrm{Zn}_{8} \mathrm{Sb}_{7}$.

This same diamond moiety is also apparent in $\mathrm{Zn}_{4} \mathrm{Sb}_{3}$. Figure 2 shows the EDD for a configuration of composition $\mathrm{Zn}_{12} \mathrm{Sb}_{10}$ (no interstitial $\mathrm{Zn}$ ). In this case, each lone $\mathrm{Sb}$ atom shares a lobe with each of two pairs of $\mathrm{Zn}$ atoms, forming zigzag chains of diamond moieties. ${ }^{13}$ The $\mathrm{Sb}$ dimers form a separate linear chain from the diamonds. The presence of interstitial $\mathrm{Zn}$, such as in $\mathrm{Zn}_{13} \mathrm{Sb}_{10}$ configurations, breaks up the chain and creates local disorder in the diamond network. High configurational entropy results because there are many possible ways for the interstitial $\mathrm{Zn}$ to distort and rearrange the diamond network, all with similar energetic consequences.

In $\mathrm{Zn}_{8} \mathrm{Sb}_{7}$, we again see the diamond moiety but with added complexity. Nonlinear chains of diamond moieties incorporate both $\mathrm{Sb}$ dimers (as in $\mathrm{ZnSb}$ ) and lone $\mathrm{Sb}$ (as in $\mathrm{Zn}_{4} \mathrm{Sb}_{3}$ ). All the diamonds are distorted out of plane, as is shown for two of the diamonds in Figure 2. The crystal structure is frustrated in the sense that it cannot settle into a single preferred ordered state, such as in $\mathrm{ZnSb}$, but like $\mathrm{Zn}_{4} \mathrm{Sb}_{3}$, there are many configurational possibilities that result in similar energetics.

One way to enumerate the possible configurational states is to consider the mismatch of symmetry between the $\mathrm{Sb}$ and $\mathrm{Zn}$ sublattices. There are six symmetrically equivalent ways to combine the higher symmetry $\mathrm{P} 6 / \mathrm{m}$ Sb sublattice with the lower symmetry $P \overline{1} \mathrm{Zn}$ sublattice. By combining these six arrangements into a single lattice, one can discover possible interstitial sites on

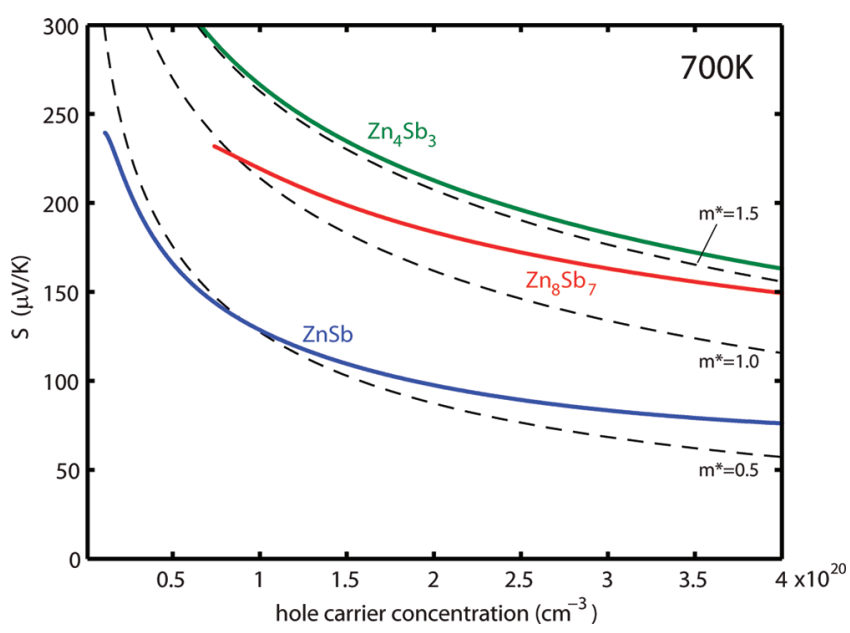

Figure 5. Calculated Seebeck coefficient as a function of (hole) carrier concentration assuming a rigid band model for $\mathrm{ZnSb}, \mathrm{Zn}_{4} \mathrm{Sb}_{3}$, and $\mathrm{Zn}_{8} \mathrm{Sb}_{7}$. Dashed lines correspond to calculations from a single parabolic band model with effective carrier mass $m^{*}$ under the constant relaxation time approximation.

the $\mathrm{Zn}$ sublattice. The combined lattice is shown in Figure 3. All the orange $\mathrm{Sb}$ sites map closely to existing sites, as expected. However, the blue Zn sites reveal many new locations. Nearly equivalent sites are combined to form an idealized lattice with 66 new possible $\mathrm{Zn}$ sites, shown in pink at the bottom of Figure 3. These sites provide a basis to probe the configurational disorder. Configurational arrangements involving $\mathrm{Zn}$ vacancies on the highly occupied blue sites and $\mathrm{Zn}$ interstitials on partially occupied pink sites can be enumerated and the energies calculated from firstprinciples calculations. This configurational disorder could likely be undetected by electron diffraction analysis due to the low occupation of certain sites. The experimentally predicted ${ }^{4}$ partial occupancy results in a composition of $\mathrm{Zn}_{29.9} \mathrm{Sb}_{28}$, which could be explained as an average of $2.1 \mathrm{Zn}$ atoms per unit cell occupying various interstitial sites. The entropy associated with this disorder will be addressed in the Phase Stability section.

Electronic Structure. The calculated electronic density of states of $\mathrm{Zn}_{8} \mathrm{Sb}_{7}$ is shown in Figure 4, along with those for $\mathrm{ZnSb}$ and $\mathrm{Zn}_{4} \mathrm{Sb}_{3}$. As expected from the Zintl-Klemm charge counting, $\mathrm{Zn}_{8} \mathrm{Sb}_{7}$ is found to be an intrinsic semiconductor like $\mathrm{ZnSb}$ and $\mathrm{Zn}_{4} \mathrm{Sb}_{3}$. The calculated band gap is comparable to that of $\mathrm{ZnSb}$ but slightly smaller than that of $\mathrm{Zn}_{4} \mathrm{Sb}_{3}$. The similar shape and magnitude of the valence band edges of $\mathrm{Zn}_{8} \mathrm{Sb}_{7}$ and $\mathrm{Zn}_{4} \mathrm{Sb}_{3}$ suggest a similar hole carrier effective mass. This result alone suggests that $\mathrm{Zn}_{8} \mathrm{Sb}_{7}$ might have electronic properties similar to those of $\mathrm{Zn}_{4} \mathrm{Sb}_{3}$. The valence band edge is composed primarily of electronic states localized around the nonbonded $\mathrm{Sb}$ atoms. The projected density of states in Figure 4 shows that the valence band is composed primarily of $\mathrm{Sb}$ p-orbital character, which is similar to previous studies of $\mathrm{Zn}_{4} \mathrm{Sb}_{3}$ and $\mathrm{ZnSb}{ }^{13}$

The calculated Seebeck coefficient, $S$, as a function of (hole) carrier concentration is shown in Figure 5 for $700 \mathrm{~K}$. This assumes a rigid band model with a carrier concentration controlled by shifting the electronic chemical potential away from the intrinsic Fermi energy. For reference, dashed lines show trends derived from a single parabolic band approximation for an effective carrier mass, $m^{*}$. The Seebeck coefficient of $\mathrm{Zn}_{8} \mathrm{Sb}_{7}$ approaches that of $\mathrm{Zn}_{4} \mathrm{Sb}_{3}$ at higher carrier concentrations for similar doping levels. An optimum carrier concentration can be 


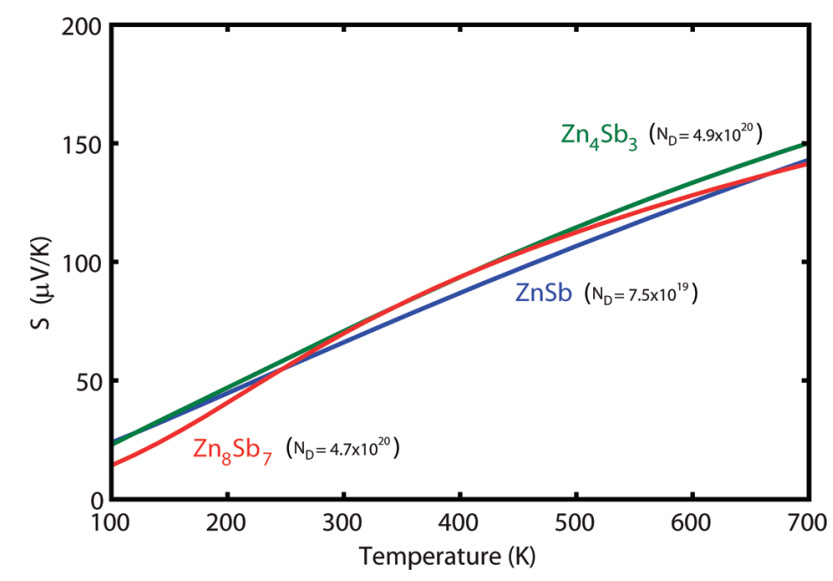

Figure 6. Calculated Seebeck coefficient as a function of temperature at constant carrier concentration (assuming extrinsic doping regime). Carrier concentration is chosen to maximize the power factors of the respective phases under the constant relaxation time approximation.

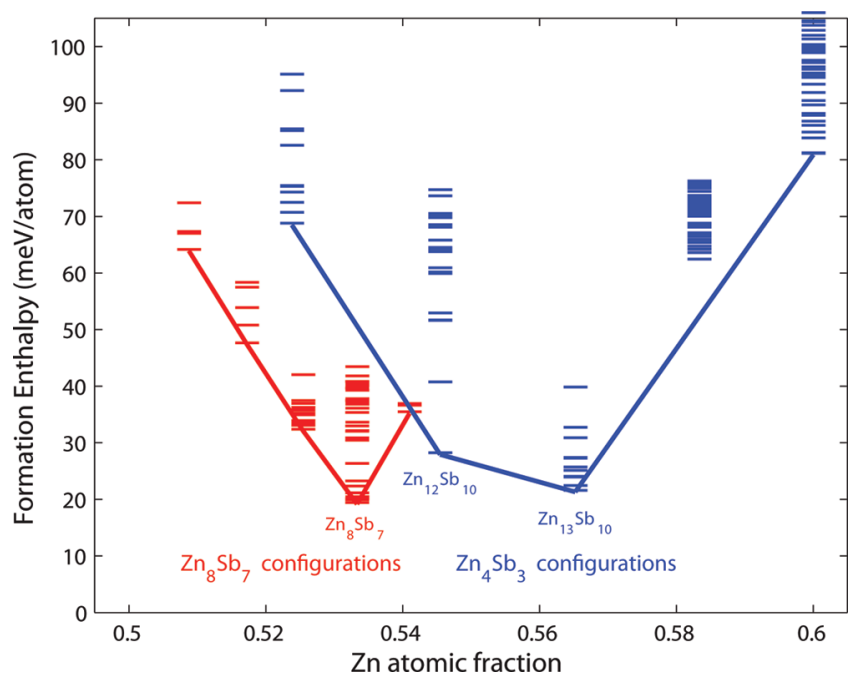

Figure 7. Formation enthalpy with respect to $\mathrm{Zn}$ and $\mathrm{ZnSb}$ at $0 \mathrm{~K}$ for $\mathrm{Zn}_{8} \mathrm{Sb}_{7}$ (red) and $\mathrm{Zn}_{4} \mathrm{Sb}_{3}$ (blue) configurations. The lines connect the respective lowest energy configurations at each composition.

chosen to maximize the power factor, $\sigma S^{2}$, with electrical conductivity, $\sigma$, calculated under the constant relaxation time approximation. An approximate relaxation time is found by fitting to experimental resistivity data for $\mathrm{ZnSb}^{25}$ and $\mathrm{Zn}_{4} \mathrm{Sb}_{3}{ }^{2}$ and assuming a similar value for $\mathrm{Zn}_{8} \mathrm{Sb}_{7}$. The constant carrier concentration Seebeck coefficient as a function of temperature is shown in Figure 6. Both $\mathrm{Zn}_{4} \mathrm{Sb}_{3}$ and $\mathrm{Zn}_{8} \mathrm{Sb}_{7}$ have a maximum power factor for a hole concentration of around $5 \times 10^{20} \mathrm{~cm}^{-3}$ and $\mathrm{ZnSb}$ for a concentration of $7.5 \times 10^{19} \mathrm{~cm}^{-3}$. It should be noted that in $\mathrm{ZnSb}$ it has proven difficult to achieve hole concentrations above $10^{19} \mathrm{~cm}^{-3}$. In $\mathrm{Zn}_{4} \mathrm{Sb}_{3}$ single-phase material, the hall carrier concentration ranges between $6 \times 10^{19}$ and $9 \times 10^{19} \mathrm{~cm}^{-3}$.

Phase Stability. Finally, we consider the thermodynamic phase stability of this new $\mathrm{Zn}_{8} \mathrm{Sb}_{7}$ phase. We note that for $\mathrm{Zn}_{8} \mathrm{Sb}_{7}$ to be thermodynamically stable, the free energy must fall below the tie-line between $\mathrm{ZnSb}$ and $\mathrm{Zn}_{4} \mathrm{Sb}_{3}$. This will prove difficult for $\mathrm{Zn}_{8} \mathrm{Sb}_{7}$, as $\mathrm{Zn}_{4} \mathrm{Sb}_{3}$ has high entropy even at room temperature. We start by considering the formation enthalpy at $0 \mathrm{~K}$ and then include entropic contributions at finite temperature.

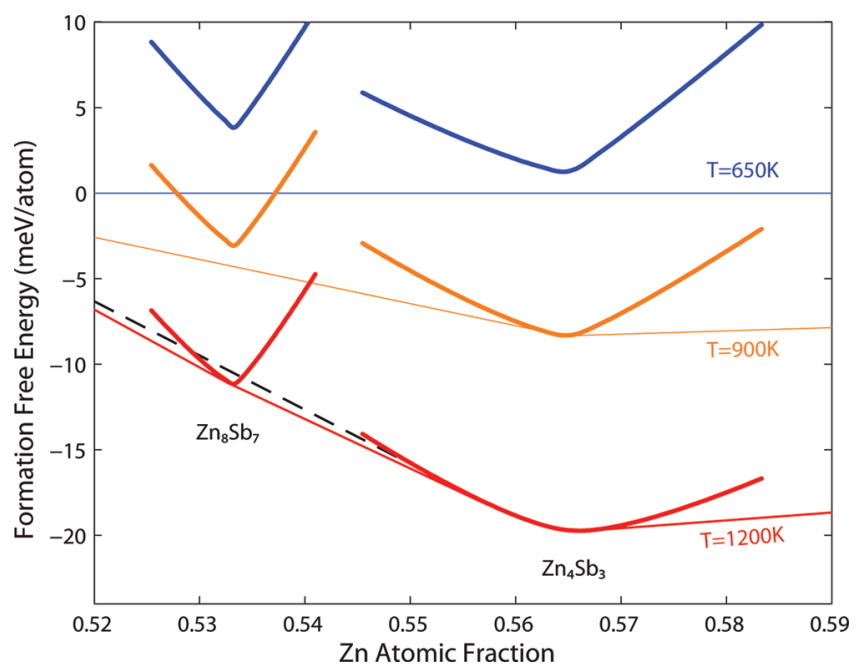

Figure 8. Calculated formation free energy curves for $\mathrm{Zn}_{4} \mathrm{Sb}_{3}$ and $\mathrm{Zn}_{8} \mathrm{Sb}_{7}$ with respect to $\mathrm{ZnSb}$ and $\mathrm{Zn}$ at three temperatures. A convex hull is drawn connecting the free energy curves of the thermodynamically stable phase at a given temperature. At $650 \mathrm{~K}, \mathrm{Zn}$ and $\mathrm{ZnSb}$ are in equilibrium over the entire composition range shown. At $900 \mathrm{~K}, \mathrm{Zn}_{4} \mathrm{Sb}_{3}$ has broken the convex hull and has a pure phase stability window on a narrow composition range. At $1200 \mathrm{~K}, \mathrm{Zn}_{8} \mathrm{Sb}_{7}$ has broken the convex hull and shows a favorable formation energy with respect to $\mathrm{ZnSb}$ and $\mathrm{Zn}_{4} \mathrm{Sb}_{3}$ (connected by the black dashed line).

Figure 7 shows the calculated formation enthalpy of $\mathrm{Zn}_{8} \mathrm{Sb}_{7}$ configurations (red) alongside those of $\mathrm{Zn}_{4} \mathrm{Sb}_{3}$ (blue). We readily observe that $\mathrm{Zn}_{8} \mathrm{Sb}_{7}$ configurations have positive formation enthalpy with respect to decomposition into $\mathrm{ZnSb}$ and $\mathrm{Zn}$. This is perhaps not unexpected due to similarities with $\mathrm{Zn}_{4} \mathrm{Sb}_{3}$, which also has positive formation enthalpy at $0 \mathrm{~K}$. However, $\mathrm{Zn}_{4} \mathrm{Sb}_{3}$ is known to be thermodynamically stable at higher temperatures ${ }^{14}$ due to both configurational and vibrational entropic contributions to the free energy. These sources might have significant contributions for $\mathrm{Zn}_{8} \mathrm{Sb}_{7}$ as well.

Entropy arises in an ensemble when many configurations are energetically accessible. It is apparent from Figure 7 that we will expect some configurational entropy in $\mathrm{Zn}_{8} \mathrm{Sb}_{7}$ at finite temperature from the several configurations with similar formation energy. Due to computational limitations on the large unit cell, we have only calculated 30 different configurations at the $\mathrm{Zn}_{32} \mathrm{Sb}_{28}$ composition as well as 30 off-stoichiometric configurations. These known configurations represent only a small subset of the possible arrangements of atoms that can be enumerated on the basis of the idealized lattice in Figure 3. Each of our known configurations is likely representative of many other configurations of similar energy. To estimate this, we consider the "mean field" entropy based on the average fractional occupancy, $x_{i}$ of each site, $i$ :

$$
S_{m f}\left[k_{\mathrm{B}} / \text { cell }\right]=-k_{\mathrm{B}} \sum_{i}\left[x_{i} \ln \left(x_{i}\right)+\left(1-x_{i}\right) \ln \left(1-x_{i}\right)\right]
$$

We can correct this value by assuming that $\mathrm{Zn}_{8} \mathrm{Sb}_{7}$ deviates from the mean field value similarly to $\mathrm{Zn}_{4} \mathrm{Sb}_{3}$. For $\mathrm{Zn}_{4} \mathrm{Sb}_{3}$, considering $90 \%$ occupancy on the framework sites and $5 \%$ occupancy on each of three interstitial sites gives an average mean field entropy of $0.48 k_{\mathrm{B}}$ /atom. This is almost twice as high as the configurational entropy calculated from the grand canonical ensemble, $0.21 k_{\mathrm{B}}$ /atom. For $\mathrm{Zn}_{8} \mathrm{Sb}_{7}$, we consider a $\mathrm{Zn}$ 
Table 1. Bulk Moduli, $B$, as Fit to the Equation of State, $E(V)$ $=\left(B / 2 V_{0}\right)\left(V-V_{0}\right)^{2}$ (for Total Energy, $E$, Unit Cell Volume, $V$, and Ground-State Volume, $\left.V_{0}\right)$, and the Room Temperature Unit Cell Volume (Calculated and Measured) for Three $\mathrm{Zn}-\mathrm{Sb}$ Phases

\begin{tabular}{lccccrc} 
& \multicolumn{2}{c}{ bulk modulus } & & \multicolumn{3}{c}{ unit cell volume $\left(\AA^{3}\right)$} \\
\cline { 2 - 3 } \cline { 6 - 7 } \cline { 5 - 7 } & $\mathrm{eV} / \AA^{3}$ & $\mathrm{GPa}$ & & DFT (GGA) & exptl & error $(\%)$ \\
$\mathrm{Zn}_{4} \mathrm{Sb}_{3}$ & 0.267 & 42.7 & & 571 & 536 & 2.1 \\
$\mathrm{Zn}_{8} \mathrm{Sb}_{7}$ & 0.240 & 38.5 & & 1577 & 1625 & -1.0 \\
$\mathrm{ZnSb}$ & 0.248 & 39.7 & & 410 & 391 & 1.6 \\
\hline
\end{tabular}

framework site occupancy of $93 \%$ based on the experimentally determined partial occupancy. The remaining $\mathrm{Zn}$ is interspersed over 66 possible interstitial sites. This results in a mean field entropy of $0.28 k_{\mathrm{B}}$ /atom, which we reduce by the correction factor for $\mathrm{Zn}_{4} \mathrm{Sb}_{3}$ to estimate the configurational entropy for $\mathrm{Zn}_{8} \mathrm{Sb}_{7}$ as $0.12 k_{\mathrm{B}}$ /atom. The GCP (eq 3) for $\mathrm{Zn}_{8} \mathrm{Sb}_{7}$ is tuned by increasing the multiplicative constants, $m_{i}$, so that, in the hightemperature limit, $S=-\partial \varphi / \partial T=0.12 k_{\mathrm{B}} /$ atom (the adjusted mean field entropy). In essence, this procedure combines enthalpy obtained from the $a b$ initio calculations with an entropy estimate based on structural similarities with the $\mathrm{Zn}_{4} \mathrm{Sb}_{3}$ phase.

The vibrational contributions to the free energy have also been accounted for in the quasiharmonic approximation. Both $\mathrm{Zn}_{4} \mathrm{Sb}_{3}$ and $\mathrm{Zn}_{8} \mathrm{Sb}_{7}$ have a favorable contribution to the free energy from phonons with respect to $\mathrm{Zn}$ and $\mathrm{ZnSb}$. In the high-temperature limit above $300 \mathrm{~K}, \mathrm{Zn}_{8} \mathrm{Sb}_{7}$ has an approximate vibrational entropy of $0.19 k_{\mathrm{B}}$ /atom, which is slightly less than that of $\mathrm{Zn}_{4} \mathrm{Sb}_{3}$, $0.22 k_{\mathrm{B}}$ /atom.

The GCP for each phase was calculated according to eq 3, and the boundary lines of phase equilibria were determined by equality of two respective GCPs. Formation free energy curves, as a function of composition, are shown for $\mathrm{Zn}_{8} \mathrm{Sb}_{7}$ and $\mathrm{Zn}_{4} \mathrm{Sb}_{3}$ at three different temperatures in Figure 8. The set of blue curves at $650 \mathrm{~K}$ correspond to the region of phase decomposition into $\mathrm{ZnSb}$ and $\mathrm{Zn}$. At the intermediate temperature in orange, the formation free energy of $\mathrm{Zn}_{4} \mathrm{Sb}_{3}$ has dropped below those of $\mathrm{Zn}$ and $\mathrm{ZnSb}$. The convex hull connects the lowest energy phase at each composition and includes the respective common tangent construction between $\mathrm{Zn}_{4} \mathrm{Sb}_{3}, \mathrm{ZnSb}$, and $\mathrm{Zn}$. At this temperature, there is no thermodynamic stability for $\mathrm{Zn}_{8} \mathrm{Sb}_{7}$, even though it is more favorable than decomposition to $\mathrm{ZnSb}$ and $\mathrm{Zn}$. At the highest temperature in red, we see that the $\mathrm{Zn}_{8} \mathrm{Sb}_{7}$ free energy curve touches the convex hull, indicating a region of phase stability. The black dashed line indicates the convex hull without the $\mathrm{Zn}_{8} \mathrm{Sb}_{7}$ phase. We see that $\mathrm{Zn}_{8} \mathrm{Sb}_{7}$ could have a temperature window of phase stability at high temperature, if there is sufficient entropy as in our estimation. We note that the calculated temperature scale does not correspond exactly to the physical Kelvin scale. Our temperatures likely overpredict the stabilization temperatures for both $\mathrm{Zn}_{4} \mathrm{Sb}_{3}$ and $\mathrm{Zn}_{8} \mathrm{Sb}_{7}$.

With our plausible assumptions for configurational entropy, we show that $\mathrm{Zn}_{8} \mathrm{Sb}_{7}$ can be thermodynamically stable at high temperatures. We also consider two possible reasons as to why $\mathrm{Zn}_{8} \mathrm{Sb}_{7}$ has only been observed experimentally in nanoparticulate form: lattice strain and surface energy. $A b$ initio calculations under the GGA typically overestimate the experimentally observed lattice parameter. This is indeed the case with $\mathrm{ZnSb}$ and $\mathrm{Zn}_{4} \mathrm{Sb}_{3}$, for which calculations predict, respectively, a $1.6 \%$ and
$2.1 \%$ lattice parameter overestimation at $300 \mathrm{~K}$ (including the calculated thermal expansion due to lattice vibrations). We would expect to see a linear trend with composition between $\mathrm{ZnSb}$ and $\mathrm{Zn}_{4} \mathrm{Sb}_{3}{ }^{26}{ }^{26}$ Contrary to expectation, the calculated lattice parameter of $\mathrm{Zn}_{8} \mathrm{Sb}_{7}$ underpredicts the experimentally characterized lattice parameter by $1.0 \%$ at $300 \mathrm{~K}$, as shown in Table 1 . This error is likely within the limits of accuracy for the experimentally characterized lattice constant, but the presence of lattice strain is possible, due to either a surface stress or other synthetic factors.

Surface effects could explain the more favorable formation of $\mathrm{Zn}_{8} \mathrm{Sb}_{7}$ in nanoparticulate form. If the surface energy of $\mathrm{Zn}_{8} \mathrm{Sb}_{7}$ was lower than the average surface energy of $\mathrm{ZnSb}$ and $\mathrm{Zn}_{4} \mathrm{Sb}_{3}$, then for a fixed number of atoms in a nanoparticle, we would expect $\mathrm{Zn}_{8} \mathrm{Sb}_{7}$ to be more favored for small particle sizes. Calculating the surface energy for each of these structures would be computationally prohibitive, but we can consider values that might be plausible. As an example, if $\mathrm{Zn}_{8} \mathrm{Sb}_{7}$ has a lower surface energy than $\mathrm{ZnSb}$ and $\mathrm{Zn}_{4} \mathrm{Sb}_{3}$ by $0.05 \mathrm{eV} / \AA^{2}\left(0.78 \mathrm{~J} / \mathrm{m}^{2}\right.$, which is on the order of differences reported in some elemental surface energies ${ }^{27}$ ), then for a $50 \mathrm{~nm}$ particle, $\mathrm{Zn}_{8} \mathrm{Sb}_{7}$ would have an energy benefit of $6 \mathrm{meV}$ /atom with respect to $\mathrm{ZnSb}$ and $\mathrm{Zn}_{4} \mathrm{Sb}_{3}$. At a particle size of $90 \mathrm{~nm}$, the surface energy difference would be negligible. Even if we assume that the surface energy is constant between all phases, we still observe that $\mathrm{Zn}_{8} \mathrm{Sb}_{7}$ has a lower bulk modulus than both $\mathrm{ZnSb}$ and $\mathrm{Zn}_{4} \mathrm{Sb}_{3}$ (Table 1). In a stressed surface condition, we expect the softer phase, $\mathrm{Zn}_{8} \mathrm{Sb}_{7}$, to have a more significant energy lowering to the surface contribution according to $P^{2} / 2 B$, where $P$ is pressure and $B$ is the bulk modulus. Thus, $\mathrm{Zn}_{8} \mathrm{Sb}_{7}$ would be slightly favored over $\mathrm{ZnSb}$ and $\mathrm{Zn}_{4} \mathrm{Sb}_{3}$. At the pressure required to adjust the calculated $\mathrm{Zn}_{8} \mathrm{Sb}_{7}$ lattice parameter to the experimental value $(-3.4 \mathrm{GPa})$, the energy correction is $3 \mathrm{meV} /$ atom in favor of $\mathrm{Zn}_{8} \mathrm{Sb}_{7}$. We suggest that through some combination of surface energy and lattice strain, $\mathrm{Zn}_{8} \mathrm{Sb}_{7}$ might be more favorable under certain conditions.

\section{CONCLUSIONS}

From our first-principles investigation of $\mathrm{Zn}_{8} \mathrm{Sb}_{7}$, we expect the theoretical bulk $\mathrm{Zn}_{8} \mathrm{Sb}_{7}$ phase to exhibit good thermoelectric properties. We predict $\mathrm{Zn}_{8} \mathrm{Sb}_{7}$ to be entropically stabilized at high temperature if our assumption of configurational entropy holds true. There are also other mechanisms, such as lattice strain or surface energy, that would favor the stability of $\mathrm{Zn}_{8} \mathrm{Sb}_{7}$ with respect to $\mathrm{ZnSb}$ and $\mathrm{Zn}_{4} \mathrm{Sb}_{3}$. The stability of $\mathrm{Zn}_{8} \mathrm{Sb}_{7}$ at high temperatures could partially explain the complexity observed in this part of the $\mathrm{Zn}-\mathrm{Sb}$ phase diagram.

\section{AUTHOR INFORMATION}

\section{Corresponding Author}

gpomrehn@caltech.edu

\section{Current Address}

${ }^{+}$Physics Department, Colorado School of Mines, Golden, Colorado 80401, United States.

\section{ACKNOWLEDGMENT}

This work is supported by the U.S. National Science Foundation via Grant No. DMR-0953378, through TeraGrid resources at NCSA and SDSC under Grant No. TGDMR050013N, the AROMURI Materials on the Brink, and the DARPA Nano Materials Program. 


\section{REFERENCES}

(1) Caillat, T.; Fleurial, J.-P.; Borshchevsky, A. J. Phys. Chem. Solids 1997, 58, 1119-1125.

(2) Toberer, E. S.; Rauwel, P.; Gariel, S.; Tafto, J.; Jeffrey Snyder, G. J. Mater. Chem. 2010, 20, 9877-9885.

(3) Snyder, G.; Christensen, M.; Nishibori, E.; Caillat, T.; Iversen, B. Nat. Mater. 2004, 3, 458-463.

(4) Birkel, C. S.; Mugnaioli, E.; Gorelik, T.; Kolb, U.; Panthöfer, M.; Tremel, W. J. Am. Chem. Soc. 2010, 132, 9881-9889.

(5) Izard, V.; Record, M.; Tedenac, J. J. Alloys Compd. 2002, 345, 257-264.

(6) Nylen, J.; Andersson, M.; Lidin, S.; Haussermann, U. J. Am. Chem. Soc. 2004, 126, 16306-16307.

(7) Nylen, J.; Lidin, S.; Andersson, M.; Iversen, B. B.; Newman, N.; Haussermann, U. Chem. Mater. 2007, 19, 834-838.

(8) Li, J.-B.; Record, M.-C.; Tedenac, J.-C. J. Alloys Compd. 2007, 438, 171-177.

(9) Liu, X.; Wang, C.; Ohnuma, I.; Kainuma, R.; Ishida, K. J. Phase Equilib. 2000, 21, 432-442.

(10) Kim, S.-G.; Mazin, I. I.; Singh, D. J. Phys. Rev. B 1998, 57, 6199-6203.

(11) Cargnoni, F.; Nishibori, E.; Rabiller, P.; Bertini, L.; Snyder, G. J.; Christensen, M.; Gatti, C.; Iversen, B. B. Chem.-Eur. J. 2004, $10,3861-3870$.

(12) Toberer, E. S.; Sasaki, K. A.; Chisholm, C. R. I.; Haile, S. M.; Goddard, W. A., III; Snyder, G. J. Phys. Stat. Sol. RRL 2007, 1, 253-255.

(13) Mikhaylushkin, A. S.; Nylen, J.; Haussermann, U. Chem.-Eur. J. 2005, 11, 4912-4920.

(14) Pomrehn, G. S.; Toberer, E. S.; Snyder, G. J.; van de Walle, A. Phys. Rev. B 2011, 83, 094106.

(15) van de Walle, A.; Asta, M. Modelling Simul. Mater. Sci. Eng. 2002, 10,521 .

(16) Perdew, J. P.; Burke, K.; Ernzerhof, M. Phys. Rev. Lett. 1996, $77,3865$.

(17) Blöchl, P. E. Phys. Rev. B 1994, 50, 17953.

(18) Kresse, G.; Furthmüller, J. Comput. Mater. Sci. 1996, 6, 15.

(19) van de Walle, A.; Asta, M.; Ceder, G. CALPHAD J. 2002, 26, 539 .

(20) van de Walle, A. CALPHAD J. 2009, 33, 266.

(21) van de Walle, A.; Ceder, G. J. Phase Equilib. 2002, 23, 348.

(22) van de Walle, A.; Ceder, G. Rev. Mod. Phys. 2002, 74, 11.

(23) Madsen, G.; Singh, D. Comput. Phys. Commun. 2006, 175.

(24) Kauzlarich, S. M. Chemistry, Structure, and Bonding of Zintl Phases and Ions; Wiley-VCH: Weinheim 1996.

(25) Bottger, P. H. M.; Pomrehn, G. S.; Snyder, G. J.; Finstad, T. G., submitted.

(26) van de Walle, A.; Ceder, G. Phys. Rev. B 1999, 59, 14992.

(27) Skriver, H. L.; Rosengaard, N. M. Phys. Rev. B 1992, 46, 7157-7168. 